\title{
High-throughput, automated quantification of white matter neurons in mild malformation of cortical development in epilepsy
}

Joan YW Liu', Matthew Ellis²,3, Hannah Brooke-Ball', Jane de Tisi', Sofia H Eriksson', Sebastian Brandner ${ }^{2,3}$, Sanjay M Sisodiya ${ }^{1,4}$ and Maria Thom ${ }^{1,2^{*}}$

\begin{abstract}
Introduction: In epilepsy, the diagnosis of mild Malformation of Cortical Development type II (mMCD II) predominantly relies on the histopathological assessment of heterotopic neurons in the white matter. The exact diagnostic criteria for mMCD II are still ill-defined, mainly because findings from previous studies were contradictory due to small sample size, and the use of different stains and quantitative systems. Advance in technology leading to the development of whole slide imaging with high-throughput, automated quantitative analysis (WSA) may overcome these differences, and may provide objective, rapid, and reliable quantitation of white matter neurons in epilepsy. This study quantified the density of NeuN immunopositive neurons in the white matter of up to 142 epilepsy and control cases using WSA. Quantitative data from WSA was compared to two other systems, semi-automated quantitation, and the widely accepted method of stereology, to assess the reliability and quality of results from WSA.
\end{abstract}

Results: All quantitative systems showed a higher density of white matter neurons in epilepsy cases compared to controls $(P=0.002)$. We found that, in particular, WSA with user-defined region of interest (manual) was superior in terms of larger sampled size, ease of use, time consumption, and accuracy in region selection and cell recognition compared to other methods. Using results from WSA manual, we proposed a threshold value for the classification of mMCD II, where $78 \%$ of patients now classified with mMCD II were seizure-free at the second post-operatively follow up.

Conclusion: This study confirms the potential role of WSA in future quantitative diagnostic histology, especially for the histopathological diagnosis of mMCD.

Keywords: mMCD, Immunohistochemistry, Quantitation, Stereology, Heterotophic, Neurons

\section{Introduction}

According to the Palmini classification, the observation of 'excess' heterotrophic neurons in layer I and the white matter are classified as mild forms of Malformation of Cortical Development (mMCD) type I and II respectively [1]. There are presently no other criteria to aid the assessment of mMCD II [2]. Despite many previous studies in this field, the oldest dating back to 1984 [3], it is still uncertain whether the density of WMN is increased in patients with epilepsy, what the threshold for

\footnotetext{
* Correspondence: m.thom@ucl.ac.uk

'Department of Clinical and Experimental Epilepsy, UCL Institute of

Neurology, Queen Square, WC1N 3BG London, UK

${ }^{2}$ Division of Neuropathology, National Hospital for Neurology and

Neurosurgery, Queen Square, WC1N 3BG London, UK

Full list of author information is available at the end of the article
}

the diagnostic confirmation of mMCD II should be [2], whether the severity of this pathological feature correlates with pre-operative MRI, and represents a clinically relevant, prognostic biomarker, in particular for outcome following epilepsy surgery [4-8]. Findings from previous quantitative studies have been contradictory [3,9-14], due to the small sample size used ( $<52$ cases), and differing counting methods, equipment, regions of analysis, and cell types studied (Additional file 1: Table S1). Excess density of WMN has also been reported in patients with schizophrenia and autism [15-19], suggesting the possibility that the WMN density may be an indicator of neurodevelopmental disorders beyond epilepsy. Consequently, there is a clinical need for the accurate, rapid evaluation of the numerical threshold of 'excess' WMN 
in epilepsy, and to determine any possible relationships between WMN density and epileptogenesis, pharmacoresistance and surgical outcome.

Recent technological advances in quantitative histology, with systems such as whole slide imaging with high-throughput, automated analysis (WSA), means that it is now possible to more accurately and efficiently quantify histological stains and immunolabelling, which may aid the clinical diagnosis of a wide range of diseases [20-24] and be instrumental in the design of personalised treatment plans which may improve patients' chances of recovery $[25,26]$. In fact, there is an increasing official acceptance of WSA: the United States Food and Drug Administration has approved the WSA of several major companies for the diagnostic evaluation/ screening of HER2, PR, ER, Ki67 and/or p53 immunolabelling (Additional file 2: Table S2), underlining the importance and relevance that quantitative histopathology has in personalised medicine. However, before such advanced system is used in routine clinical laboratory, it is important to carefully compare the cost and applicability, and validate the quality of results obtained against traditionally used methods such as semi-automated analysis, and established, gold standard approaches for quantifying the number of neurons in volume of tissue, such as stereology [27].

As such, this study aims to quantify the density of NeuN immunopositive neurons in the white matter of up to 130 samples from patients with epilepsy who have undergone a standardised surgical procedure (anterior temporal lobectomy) for the same underlying pathology (hippocampal sclerosis) and 12 controls using three quantitative methods: (i) WSA, (ii) semi-automated analysis that processes pre-acquired images of the white matter (SA), and (iii) stereology ( $n=50$ cases). This highly homogenous group of epilepsy patients has comprehensive clinical data (Additional file 3: Table S3) to allow us to investigate the clinical relevance of WMN density in epilepsy. Quantification of WMN using the three systems would also allow us to compare the quantitative results, ease of use, time consumption, reliability, and accuracy in the selection of regions and identification of cells.

\section{Material and methods}

This study was approved by the Joint Research Ethics Committee of the National Hospital for Neurology and Neurosurgery and the UCL Institute of Neurology. Patients had given consent to the use of their brain tissue in research, and the tissue was used in accordance with the current UK Human Tissue Authority guidelines. Anterior temporal lobectomy specimens from 130 epilepsy cases and 12 controls (five normal, post mortem controls with no brain pathology, and seven non-epilepsy, surgical controls) were studied. In the epilepsy cases, the resected sample was taken 1 to $1.5 \mathrm{~cm}$ from the temporal pole and the sample included the superior, middle, and inferior temporal gyrus with underlying white matter extending to the ventricle. The average age of cases with epilepsy and non-epilepsy surgical controls was 37 years and 39 years respectively. Clinical details of surgical epilepsy and control cases are summarised in Additional file 3: Table S3. Seven to $20 \mu$ m-thick, formalin-fixed, paraffin-embedded brain tissue sections from each case were processed and immunolabelled using the marker for mature neurons, Neuronal Nuclei (NeuN, 1:100, Millipore MAB377, UK), and counterstain with haematoxylin (VWR International, UK). The exact section thickness of each case (or the depth of field ' $\mathrm{z}$ ' reading) was later measured using the Zeiss Axio Imager Z2 Motorised microscope with $63 \times$ objective lens (average actual thickness \pm standard deviation, $17 \mu \mathrm{m} \pm 5$ ). NeuN immunopositive cells in the white matter of each sections were counted using (i) WSA: whole slide imaging with high-throughput, automated analytic (Tissue Studio and Developer XD, Germany) with automated and manual ROI selections, and (ii) SA: semi-automated image analytic (ImageProPlus version 6.2, Media Cybernetics, USA), and (iii) three dimensional stereology (Histometrix, Kinetic Imaging, UK).

For WSA, sections were initially scanned on a LEICA SCN400F digital slide scanner (LEICA Microsystems, Wetzlar, Germany). Scanned images were processed into a Pyramid TIFF file, stored on a fileserver and viewed and managed on the SlidePath Digital Image Hub (LEICA). Analysis was carried out with Definiens Tissue Studio 3.6 (Definiens AG Munich, Germany). Prior to the study, a set of image analysis algorithms ("solutions") with specific parameters were tested and optimised on Definiens Tissue Studio in a pilot study. The optimised parameters were applied as follows (Figure 1): First, tissue was selected from the background of each scanned image by setting homogeneity to 2.1 units, brightness to 200 units, and tissue minimum size to $450 \mu \mathrm{m}^{2}$. By setting the initialisation magnification to 0.4 units, each section was then segmented into geographical regions based on the distribution of NeuN.

The images of 142 epilepsy and control cases were categorised into groups or 'classes' with similar intensity of haematoxylin staining and NeuN immunopositive labelling so the same algorithm could be applied to all sections of the same class; this would assist and speed up the user-training process. During the user-training process in WSA with automated ROI selection, the researcher 'trained' the program to recognise the cerebral cortex, grey/white matter border, white matter and empty spaces by selecting a few segments of well-defined areas within each region from two scanned images within each 

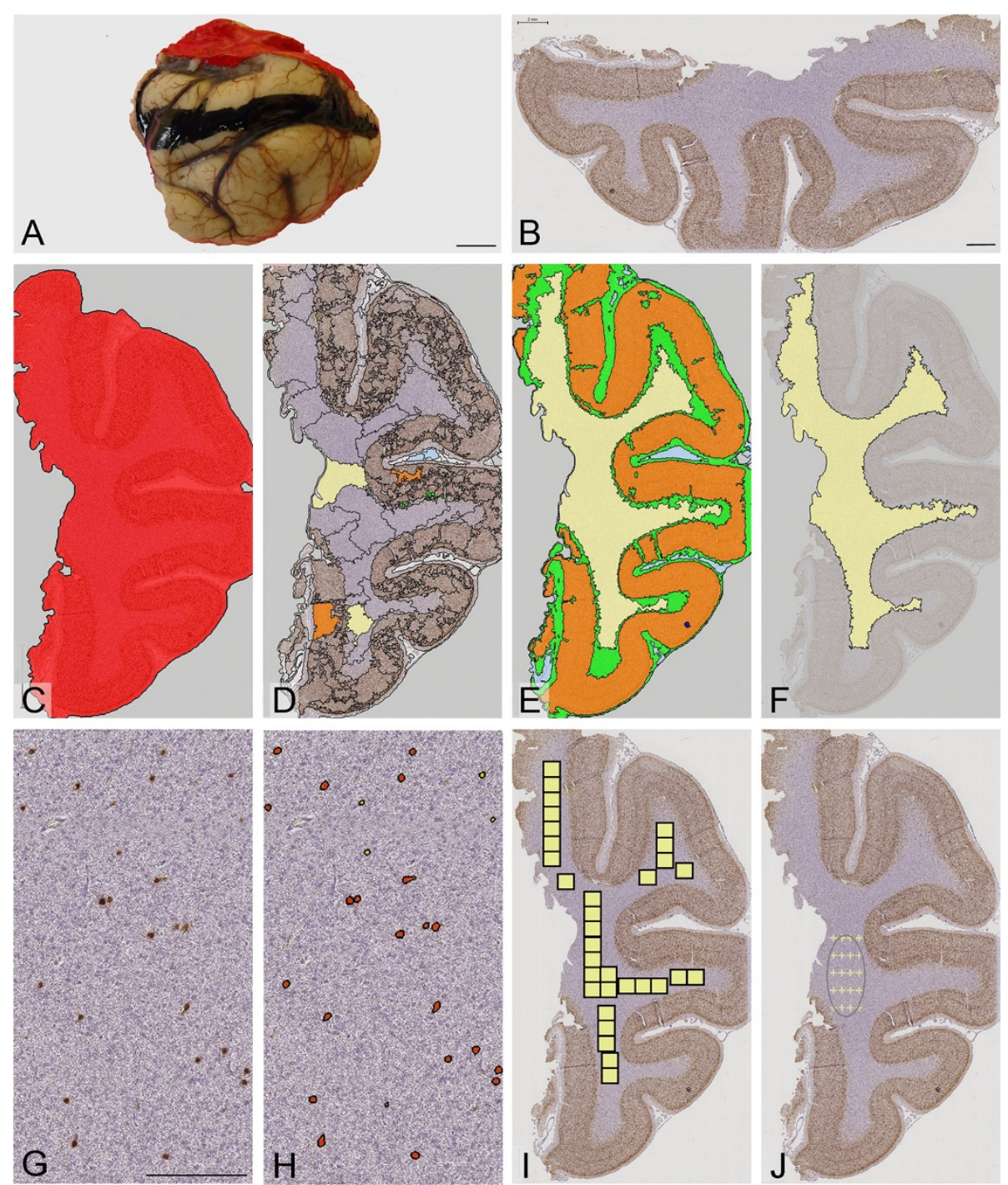

Figure 1 Quantification of NeuN immunopositive neurons in the white matter. (A) A surgically-resected temporal lobe from a patient with epilepsy who had undergone standard temporal lobectomy. (B) A $7 \mu$ m-thick section was cut, immunolabelled using the neuronal marker, NeuN, and scanned using the whole slide scanner (LEICA). Using WSA, the white matter was selected automatically or manually on digitised images of NeuN immunopositive sections. In WSA automated, the programme identified the white matter by following a series of algorithms such as tissue and background separation (C), and showed the training step, whereby the user selected 'segments' that were representative of the cortex (orange) or white matter (yellow; D), and the programme learned to identify other 'patches' of similar features (E). (F) In WSA manual, the user interactively outlined the white matter. After ROI selection, NeuN immunopositive cells in the white matter (G) of all cases were quantified by WSA (H). (I) In SA, images (yellow) were manually acquired by the user and then fed into a semi-quantitative analysis programme for quantification. (J) For stereology, a small area in the deep white matter was selected by the user and the programme moved through each point within the selection to allow the user to count NeuN immunopositive cells at 63x magnification. Scale bar = 1 cm (A), 2 mm (B-F, I-J), 200 Mm (G-H).

class. To optimise the area of white matter, filtering algorithms were applied to the grey/white matter boundary where the interface between compartments was blurred. Then the white matter was classified using the 'nearest neighbour' option, and relative area of less than 0.1 unit. These parameters/algorithms were then applied to all images in the same class.

For WSA with manual ROI selection, the researcher outlined the white matter of each image using a stylus pen on a touch-sensitive computer screen. This boundary was drawn at a set distance of $0.5 \mathrm{~mm}$ from the cortical margin, to exclude layer VI neurons. After either manual or automated ROI selection, WSA identified and counted NeuN immunopositive cells if labelled cells had an intensity of 3.1 units. All counts were expressed as cells per $\mathrm{mm}^{2}$ or $\mathrm{mm}^{3}$ adjusted by Abercrombie's correction factor to correct for section thickness and mean cell size [28]. Actual section thickness was measured on 
the Zeiss Axio Imager Z2 microscope at $63 \times$ magnification, and the cell size of NeuN immunopositive labelled cells was measured using WSA. WSA also gave the percentage of NeuN immunopositive cells that were small (area of $\left.<126.36 \mu \mathrm{m}^{2}\right)$, medium $\left(126.36-370 \mu \mathrm{m}^{2}\right)$ or large $\left(>370 \mu \mathrm{m}^{2}\right)$.

For SA, 15 images of the white matter were preacquired from each scanned image at $10 \times$ magnification. $\mathrm{NeuN}$ immunopositive cells in each image were then automatically selected by thresholding based on the intensities in red, green, and blue channels.

49 epilepsy cases and one control were quantified using stereological cell counting methods employed in our previous published studies $[11,29,30]$. The region of interest in the deep white matter of each section was first outlined at $2.5 \times$ objective on a the Zeiss Axioskop microscope linked to a stereological software (Histometrix, Kinetic Imaging, Nottingham, UK). All NeuN immunopositive cells were counted with a $63 \times$ objective using the optical dissector with uniform random field sampling, and a sampling fraction of between $25-100 \%$ to minimise coefficient of error to 0.1 unit or less where possible.

Nonparametric Kruskal-Wallis and post-hoc comparisons, Mann-Whitney, Spearman correlation, and intraclass correlation coefficient tests were performed using SPSS 20 (IBM, USA). Correlations were conducted between density of WMN and age, gender, length of seizure history, history and frequency of simple partial seizures, generalised seizures, secondary generalised seizures, and various postoperative follow-ups $\left(1^{\text {st }}\right.$ to $\left.18^{\text {th }}\right)$. To test the reliability of each method, ten randomly-selected cases (two for stereology) were re-analysed. Bland and Altman plots were presented, and Pearson's correlation and intra-class correlation coefficients using absolute agreement definition in a twoway mixed model, and single measure were calculated.

\section{Results}

The quantitative techniques and results from WSA were compared with two other quantitative systems, SA, and stereology.

\section{Area of white matter quantified}

The area of white matter sampled in 142 cases was significantly different between WSA automated and manual, SA and stereology $(\mathrm{P}<0.000)$, but not between SA and stereology $(\mathrm{P}=0.06)$. The range and average area sampled were the highest using WSA automated (246.1$6.4 \mathrm{~mm}^{2}, 74.3 \mathrm{~mm}^{2}$ ), followed by WSA manual (178.8$\left.3.4 \mathrm{~mm}^{2}, 46.7 \mathrm{~mm}^{2}\right), \mathrm{SA}\left(7.1-0.4 \mathrm{~mm}^{2}, 5.0 \mathrm{~mm}^{2}\right)$ and stereology $\left(11.9-1.2,4.0 \mathrm{~mm}^{2}\right)$ respectively; this is expected since the WSA allows the entire white matter to be sampled (Figure 1).

\section{ROI selection and identification of neurons}

For the quantification of WMN, the ability of the quantitative systems to exclude neurons in the highly-populated cortical Layer VI at the cortical/white matter demarcation from the overall WMN counts is important. In stereology, SA and WSA manual, the user manually outlined a ROI that was clearly away from the cortex/white matter demarcation, so we could be confident that only neurons in the white matter were selected (Figure 2). In WSA automated, the program was trained to exclude areas in the cerebral cortex and the grey/white matter border, and only to quantify WMN within the white matter (Figure 2). When we reviewed the screenshot images of the counted cells for quality control purposes, we found that WSA automated correctly identified and quantified WMN in most cases; there were only a few occasions where WSA automated included some neurons from the lower limits of Layer VI.
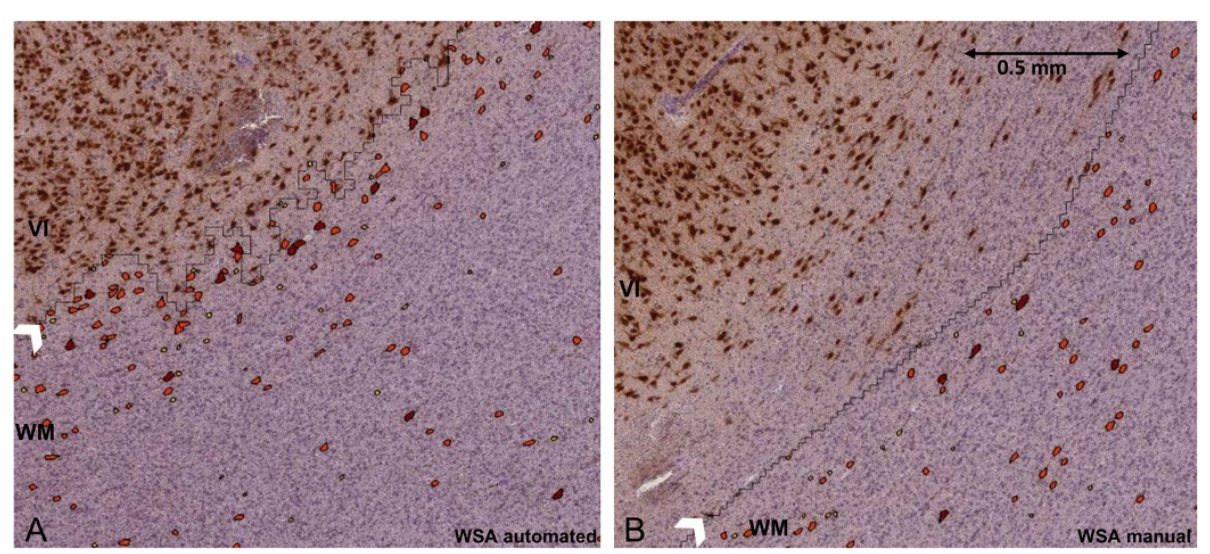

Figure 2 Cell identification using WSA automated and manual. (A) In some cases, WSA automated quantified NeuN immunopositive cells close to the grey/white matter demarcation (white arrow); some of these cells might be neurons in the cortical layer VI. (B) In contrast, because the user drew the white matter with a set distance away $(0.5 \mathrm{~mm})$ from the grey/white matter demarcation in WSA manual, we could be confident that the counted cells were in the white matter. 
NeuN immunopositive cells in all our cases were darkly labelled, and they were distinct from the background, so it was easy to train WSA and SA to accurately recognise and count these cells, and for the user to identify immunopositive cells in stereology using a $63 \times$ objective.

\section{Reliability of methods}

Ten randomly selected cases in the series (and two cases for stereology) were re-analysed to determine the reliability of each method. No significant difference in density values was observed between test and re-test $(P>0.05)$ and the Pearson correlation coefficient $(r)$ and the intraclass correlation coefficient (ICC), which are common measures of reliability [31], were above 0.6 for all methods (Figure 3A). Past studies indicate that the closer ICC and $r$ are to one, the more reliable the method [31]. In our study, WSA manual had a Pearson's correlation and an ICC value closest to one, suggesting that this method was the most reliable compared to other methods (Figure 3A-B). Bland and Altman plots also support these findings by showing good agreement with differences in most cases between test and retest values lying near zero (Figure 3B).

\section{Time consumption and ease of use}

It took an average of six hours per case to quantify WMN using stereology [29] compared to 45 minutes per case using SA, 15 and ten minutes per case using WSA automated and manual respectively (Figure $3 \mathrm{C}$ ). We found that WSA and SA were easier to use than stereology, with fewer steps involved.

\section{Density of WMN}

The WMN density of all cases, corrected with the Abercrombie principle, was significantly different between WSA automated and WSA manual, SA, and stereology $(\mathrm{P}<0.000$; Figure $4 \mathrm{~A})$, but not between WSA manual and SA $(P=0.07)$, WSA manual and stereology $(P=0.130)$, or $\mathrm{SA}$ and stereology $(\mathrm{P}=0.880)$. For epilepsy cases, the corrected WMN density was significantly different between all quantitative methods $(\mathrm{P}<0.000)$, but not between methods when applied to controls $(P=0.269)$. The lack of






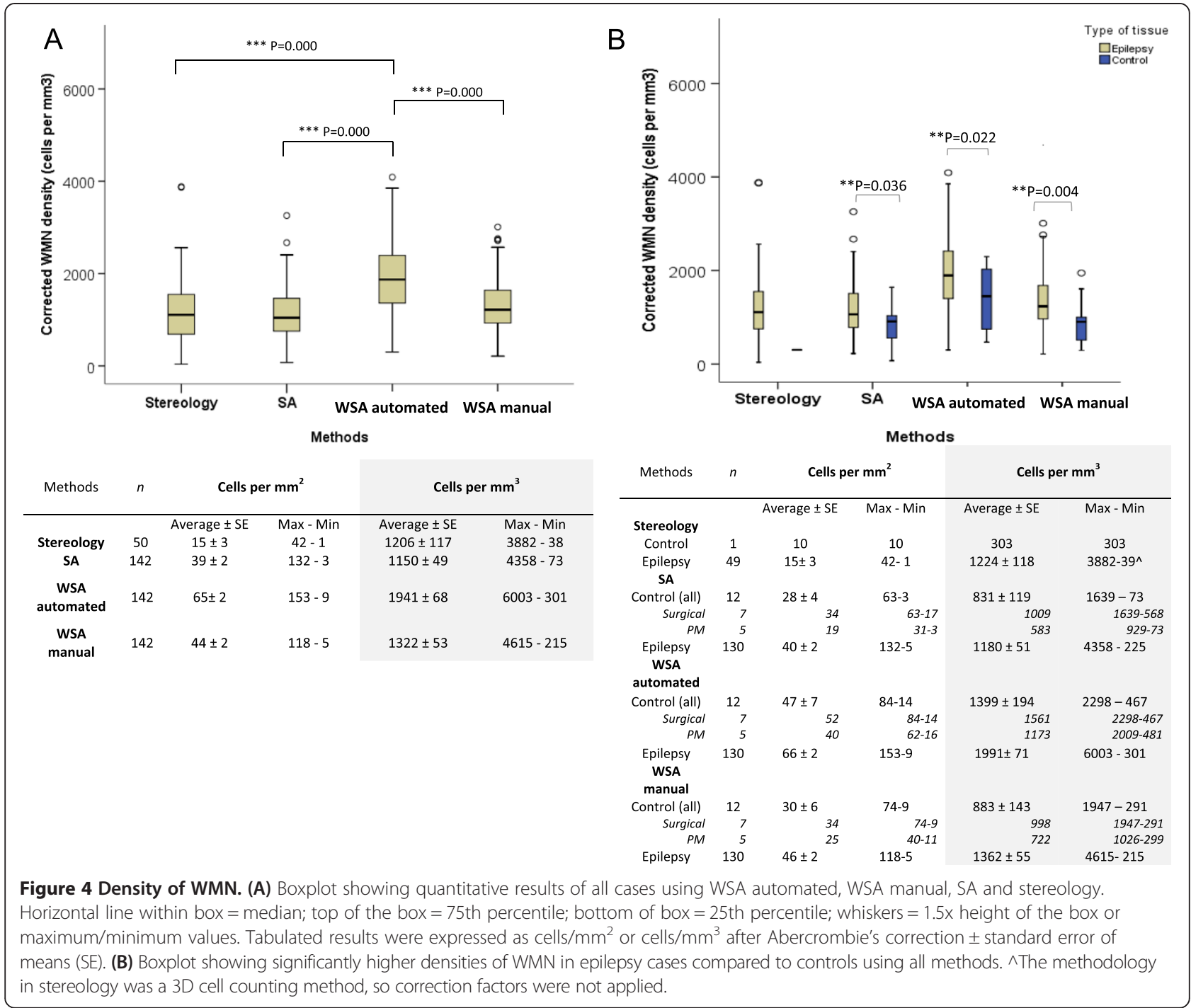

differences between methods for control samples may be due to the small number of controls analysed, or because methods were less dissimilar if there were fewer WMN in the ROI. Subsequent post-hoc comparisons showed a significant difference in the corrected WMN density in epilepsy cases between WSA automated and WSA manual, $\mathrm{SA}$, and stereology $(\mathrm{P}<0.000)$ but not between WSA manual and SA $(P=0.073)$, WSA manual and stereology $(\mathrm{P}=0.834)$, or SA and stereology $(\mathrm{P}=0.706)$. The percentages of small, medium or large NeuN immunopositive cells in epilepsy cases were not significantly different between WSA automated and manual $(\mathrm{P}>0.05)$.

\section{Comparison of WMN density between cases with epilepsy and controls}

Quantitation revealed a significantly higher corrected density of WMN in up to 130 epilepsy cases compared to up to 12 controls using WSA automated $(\mathrm{P}=0.022$;
Figure 4B), WSA manual $(\mathrm{P}=0.004)$ and $\mathrm{SA}(\mathrm{P}=0.036)$. Only one control case was analysed using stereology and statistical analysis was hence not performed. The maximum WMN density for epilepsy cases in each method were two or three-fold higher than the maximum WMN density for controls. The corrected density of WMN in control and epilepsy cases did overlap but no control case had over 2298 cells $/ \mathrm{mm}^{3}$ using WSA automated. Our controls included five post mortem, normal cases, and seven surgical, non-epilepsy cases. Surgical tissue from neurologically normal patients is by definition not available. Therefore, we used as controls histologically normal, surgically resected tissue from non-epileptic patients with traumatic brain injury or tumours. No significant difference in WMN density was observed between these groups of controls using WSA automated and manual, and SA. The corrected WMN density was significantly different between epilepsy cases and post mortem 
controls $(\mathrm{P}<0.05)$, but not against surgical non-epilepsy controls using WSA automated and manual selections, and SA. We cannot exclude that subtle oedema or mild gliosis in our surgical non-epilepsy controls may have influenced the density and location of WMN neurons in these surgical controls.

The area of white matter sampled, thickness, and average cell diameter were not significantly different between epilepsy cases and controls using any of the methods $(P>0.05)$. The percentages of small, medium, and large NeuN immunopositive cells and their average intensity measured by WSA automated were not significantly different between epilepsy cases and post mortem and surgical controls (Additional file 4: Table S4).

No significant correlation between the corrected density of WMN in epilepsy cases and clinical parameters was observed after Bonferroni correction for multiple comparison was applied at $\mathrm{P}<0.002$.

\section{Discussion}

This is the first study that has investigated and compared the reliability, accuracy, the ease of use and time consumption of three quantitative systems, WSA, SA, and stereology. We found that WSA sampled larger regions, performed faster analysis, and provided the most reproducible results compared to other systems. Using WSA, we found that a higher density of WMN in the 130 epilepsy cases compared to controls, but the density of WMN in epilepsy cases did not correlate with clinical parameters relating to seizure frequency or surgical outcome.

With the introduction of many different systems for quantitative histology, it is important that systems are rigorously tested for both reliability and cost-effectiveness, especially if results are to be used to assist in clinical diagnosis. Consequently, we compared the quantitative findings from three systems, employing three different counting approaches; stereology, SA and WSA. Threedimension stereology or design-based stereology has been long considered as the most accurate method to quantify neurons in an 'optically-defined volume of tissue' [27]. Stereology avoids the need to correct for variation in section thickness and cell size of each case as established in the Abercrombie principle [28,32], with confident, unbiased live counting of neurons at high magnification using 63 or $100 \times$ objectives. However, stereology is time-consuming, taking approximately six hours per case [29], compared to ten to 15 minutes per case with a maximally automated system such as WSA. Stereology is, therefore, impractical in a diagnostic laboratory. Findings from this study suggest that automated systems such as WSA may be a good alternative to stereology, considering that no significant difference in WMN density values was observed between stereology and WSA manual, and the results obtained from WSA manual were reproducible and reliable as evident in the intra-rater reliability coefficient tests. Furthermore, the WSA system is superior over stereology: WSA has the power to examine a large area and it avoids userselected bias of only small areas of interest (for SA and stereology) which may not be representative of the whole sample; it can also quantify sections rapidly, thus allowing for more cases and additional morphometric data to be examined without adding extra time to the count data (whereas in stereology, the use of the optical dissector method to measure the size of each cell drastically increases the analysis time). Together, these advantages support the use of WSA in quantitative histology.

Establishing an agreed numerical threshold based on the density of WMN to aid the diagnosis of MMCD II in humans is a fundamental aim for neuropathologists working in epilepsy surgical centres [2]. This information is lacking, however, mainly because previous studies have used different stains, quantitative methods, and type of specimens (see Additional file 1: Table S1; [33]). Here, using WSA on a large series of cases with uniform pathology, surgical procedure, standardised tissue processing, staining and selection of anatomical compartment, thus forming an optimal group of cases to address this research question, we found that all techniques showed a significant increase of WMN density in the epilepsy cases compared to normal post mortem controls, which was consistent with previous quantitative studies (see Additional file 1: Table S1). We found that, while the range of WMN density values from controls and epilepsy cases did overlap (consistent with $[9,11,34,35]$ ), we did not observe any control case with a corrected WMN density of over 2298 cells $/ \mathrm{mm}^{3}$ (WSA with automated selection) regardless of which quantitative method was used. Previously, Blümcke et al. suggested that a WMN density excess of 20 cells $/ \mathrm{mm}^{2}$ might be used to diagnosis mMCD II [33], but as we found that some of our normal post mortem cases had over 20 cells $/ \mathrm{mm}^{2}$ regardless of quantitative methods, we propose a scale for the certainty of the diagnosis based on results obtained using WSA with manual selection (Figure 5). For the quantitation of WMN, WSA with manual selection is faster and more consistent and reliable in identifying neurons in the white matter compared to the complete automated selection; in the manual selection, the user drew a selection that was a set distance from the grey/ white matter demarcation, therefore excluding the cortical layer VI.

The cause of the increased WMN density in epilepsy is unknown. The failure of neuronal migration, and/or the elimination or de novo generation of WMN, as either a cause or effect of seizures, have all been postulated but remain to be proven. In regards to their influence on epileptogenesis, previous studies on the functional 


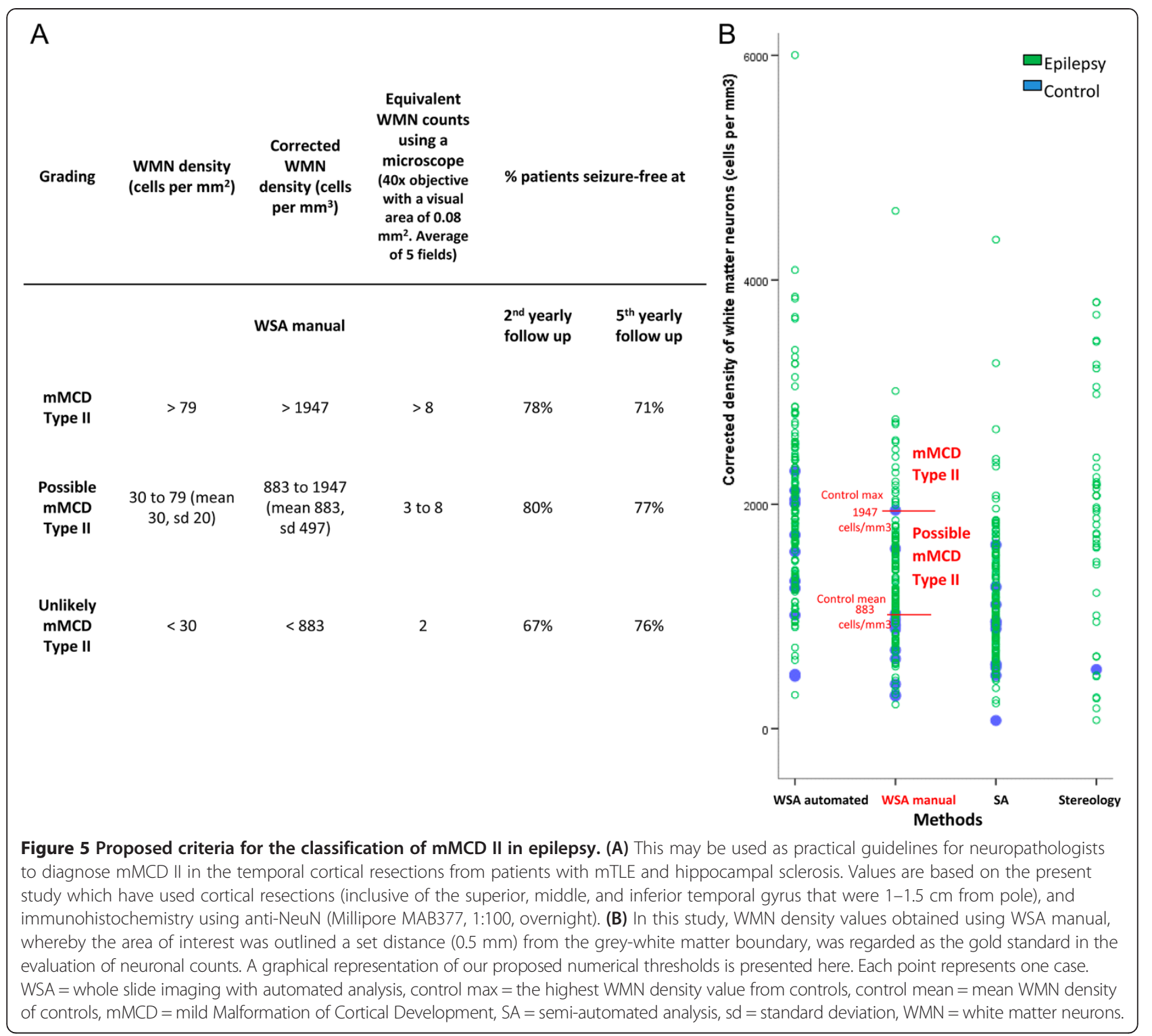

activity of WMN (normal or pathological) are limited and conflicting [5,18,36-40]. Three studies have reported that WMN density may have a predictive effect on seizure outcome following surgical resection which would support a role of increased WMN density in seizure networks in the temporal lobe $[9,11,14]$, but this finding was not observed in other similar studies [10].

\section{Conclusion}

This study showed that WSA, a modern high-throughput, automated analysis is more efficient in selecting larger regions and cells of interest, and in processing cell counts and morphometric data compared to the other systems. These obvious advantages of whole slide scanning have to be balanced with the practicalities and expense in that not every neuropathology laboratory currently has access to slide scanners and a whole slide image analysis software solution. Furthermore, users should be reminded that both WSA and SA rely on user-defined size and/or thresholding parameters to identify cells of interest, so the accuracy of quantification will ultimately be dependent on the users' settings and the quality of the acquired images/scans. Therefore, quality assurance by trained personnel after defining the parameters and repeated counts will be important. Nevertheless, the highthroughput data generated with the WSA systems can be used as a 'gold standard' to provide bench-mark criteria (Figure 5) to translate into diagnostic practice using a conventional microscope for the future assessment of mild MCDs. 


\section{Additional files}

Additional file 1: Table S1. Previous studies that have investigated WMN in adult patients with epilepsy. H\&E, Haematoxylin \& Eosin; LFB-PAS, Luxol Fast Blue- Periodic Acid Schiff; Map2, microtubule associated protein 2; NeuN, neuronal nuclei antigen; ROI, region of interest.

Additional file 2: Table S2. The US Food and Drug Administration has approved the digital whole slide imaging hardware and analytic algorithms of the above companies for the diagnostic evaluation or screening of HER2, PR, ER, Ki67 and/or p53 (Digital Pathology Associations).

Additional file 3: Table S3. Summary of clinical details from patients with epilepsy and non-epilepsy surgical controls. *Surgical outcome was classified according to International League against epilepsy (ILAE, Wieser et al. [41]) where $1=$ completely seizure free and no aura; 2 = only auras and no other seizures; 3 = one to three seizures days per year with/out auras; $4=$ four seizures days per year to $50 \%$ reduction of baseline seizure days with/out auras; $5=$ less than $50 \%$ reduction of baseline seizure days to $100 \%$ increase of baseline seizure days with/out auras; $6=$ more than $100 \%$ increase of baseline seizure days with/out auras; na $=$ unknown. $\mathrm{sd}=$ standard deviation.

Additional file 4: Table S4. Size and intensity of NeuN immunopositive cells measured using WSA automated. The percentage of NeuN immunopositive cells with an area of less than $126.36 \mu \mathrm{m} 2$ (small), between 126.36-370 $\mu \mathrm{m} 2$ (medium) and over $370 \mu \mathrm{m} 2$ (large) were not significantly different between epilepsy and control cases.

\section{Abbreviations}

WSA: Whole slide imaging with automated analysis; ICC: Intraclass correlation coefficient; mMCD: mild Malformation of Cortical Development; MRI: Magnetic resonance imaging; NeuN: Neuronal nuclei; ROI: Region of interest; SA: Semi-automated analysis; WMN: White matter neurons.

\section{Competing interests}

Authors declare that they have no competing interests.

\section{Authors' contributions}

J have made substantial contributions to the conception, design and acquisition of data, analysis and interpretation of data, and drafting of the manuscript. ME to the design and analysis of data. HB, JT, SE, SB to the acquisition and analysis of data. SS and MT to the conception and design of data, analysis and interpretation of data, and drafting of manuscript. All authors have contributed to the revising of the manuscript, and all authors have read and approved the final manuscript.

\section{Acknowledgements}

This study was supported by the Medical Health Research Council, UK (Grant number MR/J01270X/1). This work was undertaken at University College London and University College London Hospital, who receive part of their funding from the Department of Health NIHR Biomedical Centre scheme. It was also supported by the European Union Seventh Framework Programme (FP7/2007-2013) under grant agreement n602102 (EPITARGET) (SMS). We are grateful to Georgia Lockwood-Estrin for her help with stereology analyses, Lillian Martinian for her technical help in immunohistochemistry, and Lila Krishna for useful discussion on quantitative data. The WSA system, Definiens Tissue Studio, is supported by the research facility UCL IQPath.

\section{Author details}

${ }^{1}$ Department of Clinical and Experimental Epilepsy, UCL Institute of Neurology, Queen Square, WC1N 3BG London, UK. 'Division of Neuropathology, National Hospital for Neurology and Neurosurgery, Queen Square, WC1N 3BG London, UK. ${ }^{3}$ Department of Neurodegenerative Disease, UCL Institute of Neurology, Queen Square, WC1N 3BG London, UK. ${ }^{4}$ Epilepsy Society, Chesham Lane, Chalfont St Peter, SL9 ORJ Bucks, UK.

Received: 11 April 2014 Accepted: 9 June 2014

Published: 13 June 2014

\section{References}

1. Palmini A, Najm I, Avanzini G, Babb T, Guerrini R, Foldvary-Schaefer N, Jackson G, Lüders HO, Prayson R, Spreafico R, Vinters HV: Terminology and classification of the cortical dysplasias. Neurology 2004, 62(6 suppl 3):S2-S8.

2. Blümcke I, Thom M, Aronica E, Armstrong DD, Vinters HV, Palmini A, Jacques TS, Avanzini G, Barkovich AJ, Battaglia G, Becker A, Cepeda C, Cendes F, Colombo N, Crino P, Cross JH, Delalande O, Dubeau F, Duncan J, Guerrini R, Kahane P, Mathern G, Najm I, Özkara C,, Raybaud C, Represa A, Roper SN, Salamon N, Schulze-Bonhage A, Tassi L, et al: The clinicopathologic spectrum of focal cortical dysplasias: a consensus classification proposed by an ad hoc Task Force of the ILAE Diagnostic Methods Commission. Epilepsia 2011, 52(1):158-174.

3. Meencke HJ, Janz D: Neuropathological findings in primary generalized epilepsy: a study of eight cases. Epilepsia 1984, 25(1):8-21.

4. Kuzniecky R, Garcia JH, Faught E, Morawetz RB: Cortical dysplasia in temporal lobe epilepsy: magnetic resonance imaging correlations. Ann Neurol 1991, 29(3):293-298.

5. Spreafico R, Blümcke I: Focal Cortical Dysplasias: clinical implication of neuropathological classification systems. Acta Neuropathol 2010, 120(3):359-367.

6. Despotovic I, Segers I, Platisa L, Vansteenkiste E, Pizurica A, Deblaere K, Philips W: Automatic 3D Graph Cuts for Brain Cortex Segmentation in Patients With Focal Cortical Dysplasia. In Engineering in Medicine and Biology Society, EMBC, Annual International Conference of the IEEE. 2011:7981-7984.

7. Kim YH, Kang H-C, Kim D-S, Kim SH, Shim K-W, Kim HD, Lee JS: Neuroimaging in identifying focal cortical dysplasia and prognostic factors in pediatric and adolescent epilepsy surgery. Epilepsia 2011, 52(4):722-727.

8. Mühlebner A, Coras R, Kobow K, Feucht M, Czech T, Stefan H, Weigel D, Buchfelder M, Holthausen H, Pieper T, Kudernatsch M, Blümcke I:

Neuropathologic measurements in focal cortical dysplasias: validation of the ILAE 2011 classification system and diagnostic implications for MRI. Acta Neuropathol 2012, 123(2):259-272.

9. Hardiman O, Burke T, Phillips J, Murphy S, O'Moore B, Staunton H, Farrell MA: Microdysgenesis in resected temporal neocortex: incidence and clinical significance in focal epilepsy. Neurology 1988, 38(7):1041-1047.

10. Kasper BS, Stefan H, Buchfelder M, Paulus W: Temporal lobe microdysgenesis in epilepsy versus control brains. J Neuropathol Exp Neurol 1999, 58(1):22-28.

11. Thom M, Sisodiya S, Harkness W, Scaravilli F: Microdysgenesis in temporal lobe epilepsy: a quantitative and immunohistochemical study of white matter neurones. Brain 2001, 124(11):2299-2309.

12. Andres M, Andre VM, Nguyen S, Salamon N, Cepeda C, Levine MS, Leite JP, Neder L, Vinters HV, Mathern GW: Human cortical dysplasia and epilepsy: an ontogenetic hypothesis based on volumetric MRI and NeuN neuronal density and size measurements. Cereb Cortex 2005, 15(2):194-210.

13. Hildebrandt M, Pieper T, Winkler P, Kolodziejczyk D, Holthausen H, Blümcke I: Neuropathological spectrum of cortical dysplasia in children with severe focal epilepsies. Acta Neuropathol 2005, 110(1):1-11.

14. Kim DW, Lee SK, Nam H, Chu K, Chung CK, Lee S-Y, Choe G, Kim HK: Epilepsy with dual pathology: surgical treatment of cortical dysplasia accompanied by Hippocampal sclerosis. Epilepsia 2010, 51(8):1429-1435.

15. Kiehl TR, Chow EWC, Mikulis DJ, George SR, Bassett AS: Neuropathologic features in adults with 22q11.2 deletion syndrome. Cereb Cortex 2009, 19(1):153-164.

16. Connor CM, Crawford BC, Akbarian S: White matter neuron alterations in schizophrenia and related disorders. Int J Dev Neurosci 2011, 29(3):325-334.

17. Kirkpatrick B, Conley RC, Kakoyannis A, Reep R, Roberts RC: Interstitial cells of the white matter in the inferior parietal cortex in schizophrenia: an unbiased cell-counting study. Synapse 1999, 34(2):95-102.

18. Kostović I, Judaš M, Sedmak G: Developmental history of the subplate zone, subplate neurons and interstitial white matter neurons: relevance for schizophrenia. Int J Dev Neurosci 2011, 29(3):193-205.

19. Eastwood SL, Harrison PJ: Interstitial white matter neurons express less reelin and are abnormally distributed in schizophrenia: towards an integration of molecular and morphologic aspects of the neurodevelopmental hypothesis. Mol Psychiatry 2003, 8(9):821-831.

20. Apfeldorfer C, Ulrich K, Jones G, Goodwin D, Collins S, Schenck E, Richard V: Object orientated automated image analysis: quantitative and qualitative estimation of inflammation in mouse lung. Diagn Pathol 2008, 3(Suppl 1):S16.

21. Kriete A, Anderson M, Love B, Freund J, Caffrey J, Young MB, Sendera T, Magnuson S, Braughler JM: Combined histomorphometric and geneexpression profiling applied to toxicology. Genome Bio/ 2003, 4(5):R32. 
22. Warren MV, Chan WYl, Ridley JM: Analysis of protein biomarkers in human clinical tumor samples: critical aspects to success from tissue acquisition to analysis. Biomark Med 2011, 5(2):227-248.

23. Ying X, Monticello TM: Modern imaging technologies in toxicologic pathology: an overview. Toxicol Pathol 2006, 34(7):815-826.

24. Henriquez NV, Forshew T, Tatevossian R, Ellis M, Richard-Loendt A, Rogers $H$ Jacques TS, Reitboeck PG, Pearce K, Sheer D, Grundy RG, Brandner S: Comparative expression analysis reveals lineage relationships between human and murine gliomas and a dominance of glial signatures during tumor propagation in vitro. Cancer Res 2013, 73(18):5834-5844.

25. Bates S: Progress towards personalized medicine. Drug Discov Today 2010, 15(3-4):115-120.

26. Tutton $\mathrm{R}$, Jamie K: Personalized medicine in context: social science perspectives. Drug Discov Today 2014, In Press(0).

27. Schmitz C, Hof PR: Design-based stereology in neuroscience. Neuroscience 2005, 130(4):813-831.

28. Abercrombie M: Estimation of nuclear population from microtome sections. Anat Rec 1946, 94(2):239-247.

29. Eriksson SH, Free SL, Thom M, Martinian L, Sisodiya SM: Methodological aspects of $3 \mathrm{D}$ and automated $2 \mathrm{D}$ analyses of white matter neuronal density in temporal lobe epilepsy. Neuropathol Appl Neurobiol 2006, 32(3):260-270

30. Lockwood-Estrin G, Thom M, Focke NK, Symms MR, Martinian L, Sisodiya SM, Duncan JS, Eriksson SH: Correlating 3 T MRI and histopathology in patients undergoing epilepsy surgery. J Neurosci Methods 2012, 205(1):182-189.

31. Bruton A, Conway JH, Holgate ST: Reliability: what is it, and how is it measured? Physiotherapy 2000, 86(2):94-99.

32. Williams RW, Rakic P: Three-dimensional counting: an accurate and direct method to estimate numbers of cells in sectioned material. $J$ Comp Neurol 1988, 278(3):344-352.

33. Blümcke I, Vinters HV, Armstrong D, Aronica E, Thom M, Spreafico R: Malformations of cortical development and epilepsies. Epileptic Disord 2009, 11:181-193.

34. Emery JA, Roper SN, Rojiani AM: White matter neuronal heterotopia in temporal lobe epilepsy: a morphometric and immunohistochemical study. J Neuropathol Exp Neurol 1997, 56(12):1276-1282.

35. Bothwell S, Meredith GE, Phillips J, Staunton H, Doherty C, Grigorenko E, Glazier S, Deadwyler SA, O'Donovan CA, Farrell M: Neuronal hypertrophy in the neocortex of patients with temporal lobe epilepsy. J Neurosci 2001, 21(13):4789-4800.

36. Kostovic I, Rakic P: Cytology and time of origin of interstitial neurons in the white matter in infant and adult human and monkey telencephalon. J Neurocytol 1980, 9(2):219-242.

37. Shering AF, Lowenstein PR: Neocortex provides direct synaptic input to interstitial neurons of the intermediate zone of kittens and white matter of cats: a light and electron microscopic study. J Comp Neurol 1994, 347(3):433-443.

38. Avoli M, Bernasconi A, Mattia D, Olivier A, Hwa GGC: Epileptiform discharges in the human dysplastic neocortex: in vitro physiology and pharmacology. Ann Neurol 1999, 46(6):816-826.

39. Luhmann HJ, Kilb W, Hanganu-Opatz IL: Subplate cells: amplifiers of neuronal activity in the developing cerebral cortex. Front Neuroanat 2009, 3:19. doi:10.3389/neuro.05.019.2009. eCollection 2009.

40. Judaš M, Sedmak G, Pletikos M, Jovanov-Milošević N: Populations of subplate and interstitial neurons in fetal and adult human telencephalon. J Anat 2010, 217(4):381-399.

41. Wieser HG, Blume WT, Fish D, Goldensohn E, Hufnagel A, King D, Sperling MR, Lüders H, Pedley TA, ILAE Commission Report: Proposal for a new classification of outcome with respect to epileptic seizures following epilepsy surgery. Epilepsia 2001, 42(2):282-286.

doi:10.1186/2051-5960-2-72

Cite this article as: Liu et al: High-throughput, automated quantification of white matter neurons in mild malformation of cortical development in epilepsy. Acta Neuropathologica Communications 2014 2:72.

\section{Submit your next manuscript to BioMed Central and take full advantage of:}

- Convenient online submission

- Thorough peer review

- No space constraints or color figure charges

- Immediate publication on acceptance

- Inclusion in PubMed, CAS, Scopus and Google Scholar

- Research which is freely available for redistribution

Submit your manuscript at www.biomedcentral.com/submit
Ciomed Central 